\title{
Light Propagation in a Magnetic Field: Random Green Matrix Approach
}

\author{
F.A. Pinheiro ${ }^{a}$, M. RuseK $^{b}$, A. Oreowski ${ }^{b}$ \\ AND B.A. VAN TIGGELEN ${ }^{a}$ \\ ${ }^{a}$ CNRS/Laboratoire de Physique et Modélisation des Milieux Condensés \\ Université Joseph Fourier, Maison des Magistères \\ B.P. 16638042 Grenoble Cedex 9, France \\ ${ }^{b}$ Instytut Fizyki, Polska Akademia Nauk \\ al. Lotników 32/46, 02-668 Warszawa, Poland
}

(Received November 5, 2003; revised version January 20, 2004)

\begin{abstract}
We studied the spectral properties of the matrices describing multiple scattering of electromagnetic waves from randomly distributed point-like magneto-optically active scatterers under an external magnetic field $\boldsymbol{B}$. We showed that the complex eigenvalues of these matrices exhibit some universal properties such as the self-averaging behavior of their real parts, as in the case of scatterers without magneto-optical activity. However, the presence of magneto-optically active scatterers is responsible for a striking particularity in the spectra of these matrices: the splitting of the values of the imaginary part of their eigenvalues. This splitting is proportional to the strength of the magnetic field and can be interpreted as a consequence of the Zeeman splitting of the energy levels of a single scatterer.
\end{abstract}

PACS numbers: 42.25.Dd, 78.20.Ls

\section{Introduction}

Wave propagation in complex media is a broad and interdisciplinary research topic, with many applications [1-4]. Media exhibiting broken symmetry such as magneto-optical and/or chiral media, novel disordered materials such as strongly scattering semiconductor powders, and natural media such as the subsurface of the Earth are just a few examples of complex media in which wave propagation has been recently studied. In particular, the presence of magneto-optical 
activity, associated with the breaking of time-reversal symmetry and very well studied in homogeneous media [5], was shown to be at the origin of a new class of optical phenomena in inhomogeneous media. For instance, the suppression of coherent backscattering effect by the Faraday rotation was extensively studied, both experimentally [6-8] and numerically [7-9]. Theoretically, the influence of magneto-optical activity on the coherent backscattering cone was investigated by MacKintosh and John [10], van Tiggelen et al. [11] and Lacoste and van Tiggelen [12]. It was theoretically suggested [13] that the Faraday effect generates a magneto-transverse light diffusion in random media similar to the electronic Hall effect. This so-called photonic Hall effect, which finds its origin in light scattering by one single Faraday-active scatterer, was recently observed [14, 15].

In spite of these extensive studies over the last years, the impact of magneto-optical effects on some open and fundamental phenomena is still unknown. The study of the onset of Anderson or strong localization of light is one of them. Anderson localization refers to an inhibition of wave transport in disordered media due to the interference of multiple scattered waves [16]. Anderson localization of light was recently reported in semiconductor powders [17] and disagreement even exists without magnetic fields [18]. As an alternative, it was realized that clouds of laser-cooled atoms constitute a promising scattering media in which Anderson localization of light could be achieved [19]. In fact, they constitute a perfectly monodisperse and non-absorbing system of resonant point-like scatterers with large cross-sections and huge magneto-optical effects [20]. A deeper understanding of the onset of the localized regime in such media, including the role of magneto-optical activity, is thus needed and this constitutes the main motivation for the present work. This motivation was stimulated by the first experiments on multiple light scattering in cold atomic clouds [21], including the observation of the coherent backscattering effect in cold rubidium [21] and strontium [22]. Especially for strontium, mimicking a two-level atom near the frequency where the experiments have been conducted, should be very well described by the present study.

We will investigate electromagnetic wave propagation in magneto-optically active media within the framework of the ab initio approach introduced earlier by Rusek and Orłowski [23]. This approach is based on a study of the spectra of the Green matrices describing wave scattering from an assembly of randomly distributed point-like dipoles (i.e., particles much smaller than the wavelength of light), and has been employed to investigate Anderson localization of scalar waves in the case of a 2D system [24] with nontrivial boundary conditions [25]. It was shown that the spectra of these random Green matrices exhibit some universal properties, such as the "clustering" of the real part of their eigenvalues emerging in the limit of an infinite medium, which was interpreted as the appearance of localized states $[23,26]$. In the present paper we modify this method in order to include the magneto-optical activity inside the dipoles. 


\section{T-matrix in the presence of a magnetic field}

In the following, we deal with classical magneto-optical dipoles located in vacuum. The $3 \times 3 T$-matrix $\boldsymbol{t}(k)$ describes the light scattering by one particle and exhibits a scattering resonance at frequency $\omega_{0}$ with line width $\Gamma\left(k=\omega / c_{0}\right.$ is the wave number) [11].

All multiple scattering processes in an assembly of $N$ magneto-optical scatterers situated at the positions $\boldsymbol{r}_{1}, \boldsymbol{r}_{2}, \ldots, \boldsymbol{r}_{N}$ are fully described by the $3 N \times 3 N$ $M$ matrix [23-27]:

$$
\boldsymbol{M}(k) \equiv \boldsymbol{T}(k) \cdot[\boldsymbol{U}-\boldsymbol{G}(k) \cdot \boldsymbol{T}(k)]^{-1},
$$

where $\boldsymbol{U}$ is the $3 N \times 3 N$ unit matrix, $\boldsymbol{T}(k)$ is a $3 N \times 3 N$ matrix which has $3 \times 3$ diagonal blocks equal to $t(k)$ and zeroes otherwise, and the $3 \times 3$ blocks of the $3 N \times 3 N$ G-matrix are given by

$$
\boldsymbol{G}_{n m}(\omega)= \begin{cases}-\frac{\exp \left(\mathrm{i} k r_{n m}\right)}{\mathrm{i} 4 \pi r_{n m}}\left\{\left(\boldsymbol{U}-\widehat{\boldsymbol{r}}_{n m} \widehat{\boldsymbol{r}}_{n m}\right)\right. & \\ \left.-\left(\frac{1}{\mathrm{i} k r_{n m}}+\frac{1}{\left(k r_{n m}\right)^{2}}\right)\left[\boldsymbol{U}-3 \widehat{r}_{n m} \widehat{\boldsymbol{r}}_{n m}\right]\right\} & \text { for } n \neq m, \\ 0 & \text { for } n=m\end{cases}
$$

$(m, n=1,2, \ldots N) . \boldsymbol{G}_{n m}$ may be understood as an electric Green function of the Maxwell equations calculated from the relative positions of the $N$ dipoles [28].

The $3 \times 3$ total $\boldsymbol{T}$-matrix is given by

$$
\boldsymbol{T}_{\boldsymbol{k}, \boldsymbol{k}^{\prime}}=\sum_{m=1}^{N} \sum_{n=1}^{N} \mathrm{e}^{-\mathrm{i} \boldsymbol{k} \cdot \boldsymbol{r}_{m}} \boldsymbol{M}_{m n} \mathrm{e}^{\mathrm{i} \boldsymbol{k}^{\prime} \cdot \boldsymbol{r}_{n}},
$$

where $\boldsymbol{M}_{m n}$ is the $m n$-th $3 \times 3$ block of the $3 N \times 3 N$ matrix $\boldsymbol{M}$, and $\boldsymbol{k}$ and $\boldsymbol{k}^{\prime}$ are, respectively, the incident and the scattered wave vectors, and in the far field $\left|k^{\prime}\right|=|k|=k=\omega / c_{0}$.

Equation (3) determines the electric field scattered by the magneto-optical dipoles. It requires the diagonalization of the $\boldsymbol{M}$-matrix (1).

The $t$-matrix describing one single scatterer $\boldsymbol{t}(\omega)$ can be expressed as a Born series [28]:

$$
\boldsymbol{t}(\omega)=\boldsymbol{V}(\omega)+\boldsymbol{V}(\omega) \cdot \boldsymbol{G}_{0}(\omega) \cdot \boldsymbol{V}(\omega)+\ldots=\left[\frac{1}{\boldsymbol{V}(\omega)}-\boldsymbol{G}_{0}(\omega)\right]^{-1},
$$

where $\boldsymbol{V}(\omega)$ is the optical potential for a point scatterer and $\boldsymbol{G}_{0}(\omega)$ is the return Green function, i.e., the dyadic Green function $\boldsymbol{G}_{0}(\omega, \boldsymbol{r})$ evaluated at $\boldsymbol{r}=\mathbf{0}$. The optical potential is, in contrast to the case of quantum mechanical scattering theories, frequency-dependent and for a dipole at the position $\boldsymbol{r}_{i}$ subject to an external magnetic field $\boldsymbol{B}$ it takes the form

$$
\boldsymbol{V}(\omega, r)=-\left(\frac{\omega}{c_{0}}\right)^{2} \alpha(\boldsymbol{B}) \delta\left(\boldsymbol{r}-\boldsymbol{r}_{i}\right)
$$


where $\alpha(\boldsymbol{B})$ is the polarizability tensor of the scatterer. It has the dimension of a volume which represents the microscopic size of the dipoles and is typically of the order of $a_{0}^{3}$ ( $a_{0}$ - the Bohr radius), but can be much bigger when the Rydberg states are involved in the transition. For a magneto-optical dipole the polarizability $\alpha(\boldsymbol{B})$ is given by

$$
\frac{1}{\alpha(\boldsymbol{B})}=\frac{\boldsymbol{U}}{\alpha_{0}}+\mathcal{L}(\boldsymbol{B})
$$

where $\alpha_{0}$ is the ordinary polarizability of the scatterer [29] and $\mathcal{L}(\boldsymbol{B})$ is a magneto-optical correction. We shall restrict ourselves to linear orders in $\boldsymbol{B}$, in which case this correction is given by [11]:

$$
\mathcal{L}_{i j}(\boldsymbol{B})=\beta(B) \mathrm{i} \epsilon_{i j k} \widehat{B}_{k},
$$

where $\mathrm{i} \epsilon_{i j k} \widehat{B}_{k}$ is an antisymmetric Hermitian tensor in terms of the Levi-Civita tensor $\epsilon_{i j k}, B=|\boldsymbol{B}|$, and $\widehat{B}=\boldsymbol{B} / B$. The dimensionless quantity $\beta(B)$ is defined by $\beta \equiv \frac{4 \pi c^{3}}{\alpha_{0} \omega_{0}^{3}} \frac{V B}{\Gamma}\left(\frac{c}{\omega_{0}}\right)^{2}$, with $V$ the Verdet constant of the scatterer [11].

Equation (2) shows that the matrix elements of the return Green function $\boldsymbol{G}_{0}(\omega)$ exhibit singularities at $r=0$. This feature can be handled by regularization [29]:

$$
\tilde{\boldsymbol{G}}_{0}(k, r=0)=\left(\frac{\Lambda}{6 \pi}+\mathrm{i} \frac{k}{6 \pi}\right) \boldsymbol{U} .
$$

The inverse length scale $A$ defines $\omega_{0}$ and $\Gamma$ by the relations $1 / A=\left(\omega_{0} / c\right)^{2}\left(\alpha_{0} / 6 \pi\right)$ and $1 / \Lambda=\left(\Gamma c / \omega_{0}^{2}\right)$, respectively. Using Eqs. (8) and (4), we can write

$$
\boldsymbol{t}^{-1}(\omega)=\boldsymbol{V}^{-1}(\omega)-\boldsymbol{G}_{0}(\omega)=\frac{\Lambda}{6 \pi}\left(1-\frac{\omega_{0}^{2}}{\omega^{2}}\right) \boldsymbol{U}+\mathrm{i} \frac{\omega}{6 \pi c}-\frac{c^{2}}{\omega_{0}^{2}} \mathcal{L}(\boldsymbol{B}),
$$

where Eqs. (5), (6) have been used. Assuming that $\omega_{0} \Gamma \ll 1$ (for an atom, typically $\omega_{0} \Gamma \approx 10^{-6}$ ) we can put, close to the resonance, $\omega \approx \omega_{0}$, and introduce the detuning $\Delta \equiv\left(\omega-\omega_{0}\right) / \Gamma$. We can rewrite the total scattering matrix $M(\omega)$ in Eq. (1) as

$$
\boldsymbol{M}(\omega)=\frac{4 \pi c_{0}}{\omega_{0}}\left[\boldsymbol{U} \Delta+\frac{2}{3} \boldsymbol{U}_{\mathbf{i}}-\frac{4 \pi c_{0}}{\omega_{0}} \boldsymbol{G}\left(\omega_{0}\right)-\mathcal{L}(\boldsymbol{B})\right]^{-1}
$$

Let us notice that the only frequency dependence of the total scattering matrix (10) near the resonance is contained in $\Delta$. Equation (10) shows that to find the $\boldsymbol{T}$-matrix in Eq. (3), we need to diagonalize the matrix

$$
\boldsymbol{G}_{\mathrm{MO}}\left(\omega_{0}\right)=\frac{4 \pi c}{\omega_{0}} \boldsymbol{G}\left(\omega_{0}\right)+\mathcal{L}(\boldsymbol{B})
$$

This matrix is independent of the frequency $\omega$ and the presence of the second term in (11) makes the difference with previous works [23-27].

In the following section, we will numerically diagonalize the matrix $\boldsymbol{G}_{\mathrm{MO}}$ for an arbitrary number of scatterers and investigate the consequences of the magneto-optical activity for the distribution of its eigenvalues. 


\section{Results and discussion}

As an example of a typical distribution of the eigenvalues $\lambda$ of the randomly chosen $\boldsymbol{G}_{\mathrm{MO}}$ matrix, in Fig. 1 we plot the real and the imaginary parts of the spectrum of $\boldsymbol{G}_{\mathrm{MO}}$ for a configuration of $N=1000$ resonant dipoles in a sphere, with the uniform density $\rho=1$ scatterer per wavelength cubed $\lambda_{0}^{3}$. This situation resembles the case of scalar scatterers [23-27]. We observe that eigenvalues tend to "cluster" themselves around the line $\operatorname{Re} \lambda=-1$. Previous numerical work has shown that this kind of behavior appears by increasing the size of the system [23-27]. This fact suggests that in the limit of an infinite system all the eigenvalues will move toward the value $\operatorname{Re} \lambda=-1$, a transition that may be interpreted with the formation of a band of localized states [23-27].

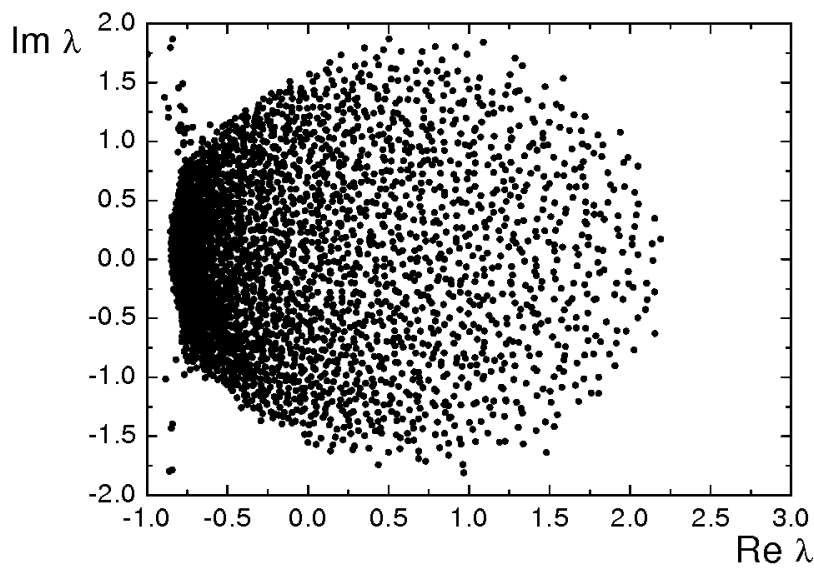

Fig. 1. Spectrum of the $\boldsymbol{G}_{\mathrm{MO}}$ matrix corresponding to an arbitrary configuration of $N=1000$ point-like scatterers calculated with a vanishing value of the external magnetic field. This situation corresponds to the case of scatterers without magneto-optical activity.

The impact of the magneto-optical activity on the spectra of the matrix $\boldsymbol{G}_{\mathrm{MO}}$ can be seen in Fig. 2 where we plot the real and the imaginary parts of $\lambda(\boldsymbol{B})$ calculated with the same parameters as in Fig. 1 but now with a nonzero value of the external magnetic field $(\beta(B)=5)$. We can observe not only the tendency of the eigenvalues to "cluster" themselves around the line $\operatorname{Re} \lambda=-1$, as in the case of scatterers without magneto-optical activity, but also a striking consequence of the magneto-optical activity: the splitting of the values of the imaginary part $\operatorname{Im} \lambda$ of the eigenvalues. In order to explain this result, one should recall the physical meaning of the real and imaginary parts of the eigenvalues of the $\boldsymbol{G}_{\mathrm{MO}}$ matrix. Within our model for each single scatterer, which exhibits an internal scattering resonance (also called the Breit-Wigner type resonance), the real and imaginary parts of the eigenvalues correspond approximately to the relative width and to the 


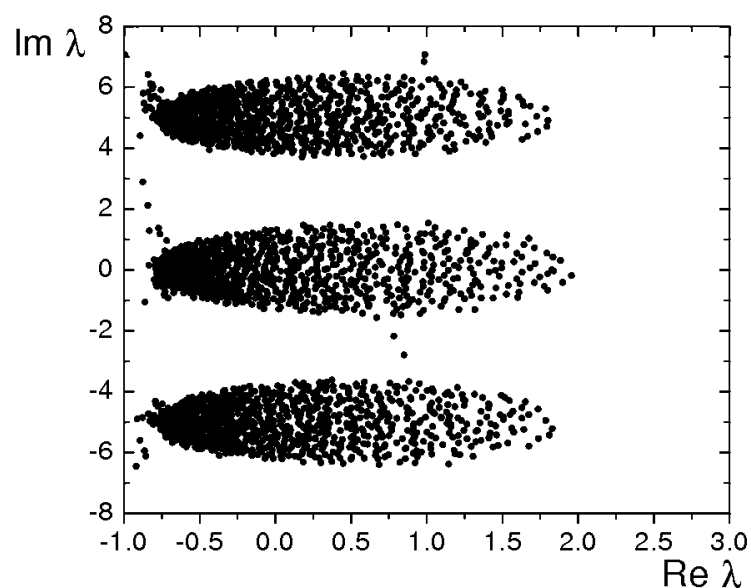

Fig. 2. As in Fig. 1, but now with a non-vanishing value of the external magnetic field. The value of the dimensionless magnetic field strength parameter is $\beta(B)=5.0$. Let us notice the splitting of the values of the imaginary parts of the eigenvalues, which is absent in the case of scatterers without magneto-optical activity exhibited in Fig. 1.

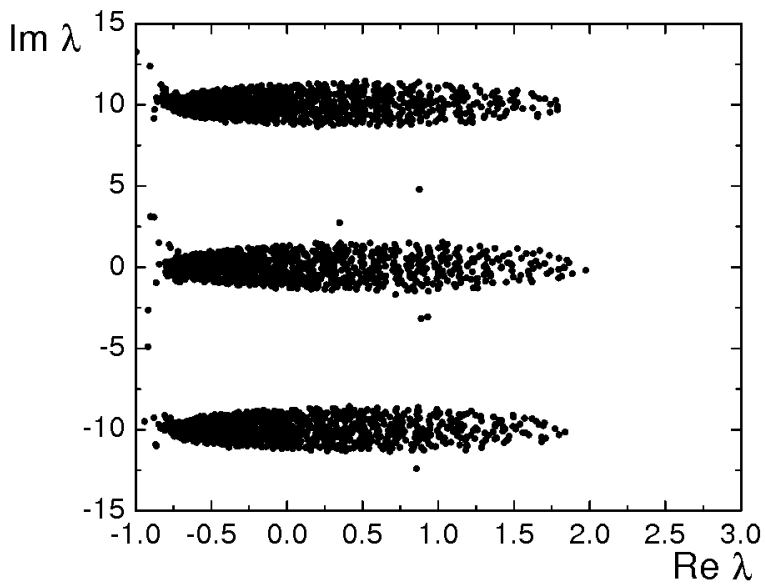

Fig. 3. As in Fig. 2, but now with a value of the magnetic field strength parameter twice larger, i.e., $\beta(B)=10.0$.

positions of the resonances, respectively [27]. As a result, the observed splitting of the values of $\operatorname{Im} \lambda$ can be interpreted as a consequence of the Zeeman splitting of the energy levels of each scatterer induced by the application of an external magnetic field.

This interpretation is confirmed by the results shown in Fig. 3, where we have calculated the spectra of the $\boldsymbol{G}_{\text {MO }}$ matrix for the same number of scatterers and parameters used in the previous figures but now with a magnetic field strength parameter $\beta(B)$ twice larger than in Fig. 2, i.e., $\beta(B)=10$. By comparing the 
positions where the values of $\operatorname{Im} \lambda$ tend to distribute themselves with the ones in Fig. 2, we notice that they are effectively linearly proportional to the magnetic field strength parameter $\beta(B)$. This fact confirms that the observed splitting of the values of $\operatorname{Im} \lambda$ actually reflects a genuine Zeeman splitting of the energy levels of a single scatterer. In addition, we observe that for a larger value of the magnetic field, the distribution of the values of $\operatorname{Im} \lambda$ around their split positions tends to be less pronounced.



Fig. 4. Surface plot of the density of eigenvalues $P(\lambda)$ calculated for 100 distinct configurations of $N=300$ point-like scatterers. The value of the magnetic field strength parameter is $\beta(B)=5.0$.

In Fig. 4 we plot the probability distribution $P(\lambda)$ calculated from 100 distinct realizations of the disorder of 300 scatterers distributed in a sphere with uniform density $\rho=1$ and subject to an external magnetic field. The probability distribution $P(\lambda)$ was normalized by $\int \mathrm{d}^{2} \lambda P(\lambda)$. The inspection of this plot reveals that values of $\operatorname{Im} \lambda$ are approximately equipartitioned between the three split positions in the $\operatorname{Im} \lambda$ axis induced by the external magnetic field. Furthermore, $P(\lambda)$ tends to move towards the $\operatorname{Re} \lambda=-1$ line, as observed in the case of scatterers without magneto-optical activity [27]. This "clustering" behavior of the spectra of the random Green matrices, which we now also verify when the form of these matrices is modified by the presence of an external magnetic field, seems to be, as pointed out by Rusek et al. [27], a truly universal behavior for random Green matrices for which some simple underlying explanation argument should exist.

\section{Summary and conclusion}

In conclusion, we have investigated the properties of the matrices that describe multiple scattering of electromagnetic waves by the Faraday active dipoles 
subject to an external magnetic field. These matrices can be expressed as a sum of the complex-symmetric random Green matrix, whose elements are equal to the free-space Green function calculated from the positions of each pair of scatterers, and a block-diagonal matrix, linearly proportional to the magnetic field, which is associated with the magneto-optical correction. We have shown that the spectra of these matrices exhibit the same universal property as in the absence of magneto-optical activity, which is the "clustering" of the real part of their eigenvalues around the line $\operatorname{Re} \lambda=-1$. However, the presence of an external magnetic field modifies the spectra in a striking way: it induces a splitting of the values of the imaginary part of the eigenvalues. This splitting is proportional to the strength of the magnetic field. Physically, since the imaginary part of the spectra approximately corresponds to the locations of the resonances of a single scatterer, this splitting of the spectra can be interpreted as a genuine Zeeman splitting of the energy levels of a single scatterer. We think that results presented here constitute an important step for the study of the phenomenon of the Anderson localization of light using the framework of the random Green matrix method. In order to unambiguously study the onset of the localized regime in media subject to magnetic fields, one should investigate further aspects of light propagation in such media, such as, for instance, the pattern of the coherent backscattering cone. The study of the backscattering cone in such a medium can provide some predictions about the magnitude of the transport mean free path in the localized regime. These predictions could be experimentally verified, for instance, in clouds of cold atoms exhibiting important magneto-optical effects. These aspects are under investigation and will be reported soon.

\section{Acknowledgments}

This work was supported by the State Committee for Scientific Research (Poland) under grant no. 2 P03B 04419.

\section{References}

[1] Scattering and Localization of Classical Waves in Random Media, Ed. Ping Sheng, World Scientific, Singapore 1990.

[2] New Aspects of Electromagnetic and Acoustic Wave Diffusion, Eds. POAN research Group, Springer-Verlag, Heidelberg 1998.

[3] Diffuse Waves in Complex Media, Ed. J.P. Fouque, Kluwer, Dordrecht 1999.

[4] Wave Diffusion through Complex Media, Ed. P. Sebbah, Kluwer, Dordrecht 2001.

[5] L.D. Barron, Molecular Light Scattering and Optical Activity, Cambridge University Press, Cambridge 1982.

[6] F.A. Erabacher, R. Lenke, G. Maret, Europhys. Lett. 21, 551 (1993).

[7] R. Lenke, R. Lehner, G. Maret, Europhys. Lett. 52, 620 (2000). 
[8] R. Lenke, G. Maret, Eur. Phys. J. B 17, 171 (2000).

[9] A.S. Martinez, R. Maynard, Phys. Rev. B 50, 3714 (1994).

[10] F.C. MacKintosh, S. John, Phys. Rev. B 37, 1884 (1988).

[11] B.A. van Tiggelen, R. Maynard, Th.M. Nieuwenhuizen, Phys. Rev. E 53, 2881 (1996).

[12] D. Lacoste, B.A. van Tiggelen, Phys. Rev. E 61, 4556 (2000).

[13] B.A. van Tiggelen, Phys. Rev. Lett. 75, 422 (1995).

[14] G.L.J.A. Rikken, B.A. van Tiggelen, Nature (London) 381, 54 (1996).

[15] G. Düchs, A. Sparenberg, G.L.J.A. Rikken, B.A. van Tiggelen, Phys. Rev. E 62, $2840(2000)$.

[16] P.W. Anderson, Phys. Rev. 109, 1492 (1958).

[17] D.S. Wiersma, P. Bartolini, A. Lagendijk, R. Righini, Nature (London) 390, 671 (1997).

[18] F. Scheffold, R. Lenke, R. Tweer, G. Maret, Nature (London) 398, 207 (1999).

[19] Th.M. Nieuwenhuizen, A.L. Burin, Yu. Kagan, G.V. Shlyapnikov, Phys. Lett. A 184, $360(1994)$

[20] G. Labeyrie, C. Miniatura, R. Kaiser, Phys. Rev. A 64, 033402-1 (2001).

[21] G. Labeyrie, F. de Tomasi, J.-C. Bernard, C.A. Müller, C. Miniatura, R. Kaiser, Phys. Rev. Lett. 83, 5266 (1999); G. Labeyrie, F. de Tomasi, J.-C. Bernard, C.A. Müller, C. Miniatura, R. Kaiser, J. Opt. B: Quantum Semiclassical Opt. 2, $672(2000)$.

[22] Y. Bidel, B. Klappauf, J.-C. Bernard, D. Delande, G. Labeyrie, C. Miniatura, D. Wilkowski, R. Kaiser, Phys. Rev. Lett. 88, 203902 (1999).

[23] M. Rusek, A. Orłowski, Phys. Rev. E 51, R2763 (1995).

[24] M. Rusek, A. Orłowski, J. Mostowski, Phys. Rev. E 56, 4892 (1997).

[25] M. Rusek, A. Orłowski, Phys. Rev. E 59, 3655 (1999).

[26] M. Rusek, A. Orłowski, J. Mostowski, Phys. Rev. E 53, 4122 (1996).

[27] M. Rusek, J. Mostowski, A. Orłowski, Phys. Rev. A 61, 022704 (2000).

[28] A. Lagendijk, B.A. van Tiggelen, Phys. Rep. 270, 143 (1996).

[29] P. de Vries, D.V. van Coevorden, A. Lagendijk, Rev. Mod. Phys. 70, 447 (1998). 